\title{
A fuzzy clustering-based method for scenario analysis in strategic planning: The case of an Asian pharmaceutical company
}

\author{
M.S. Pishvaee and M. Fathi \\ Department of Industrial Engineering, Amirkabir University of Technology, \\ Tehran, Iran \\ ms-pishvaee@aut.ac.ir and mfathi_ieyahoo.com \\ F. Jolai* \\ Department of Industrial Engineering, Faculty of Engineering, \\ University of Tehran, Iran \\ fjolai@ut.ac.ir \\ Received August 2008
}

\begin{abstract}
In today's rapid changing market situations, many nations and companies try to keep or make better their situation and gain more market share by creating competitive advantages. Because of growing number of uncertain parameters in the environment and lack of information about the future, the strategic choice has become very complex and critical. One of the popular tools for solving the problem is scenario analysis. In this paper based on fuzzy clustering we propose a method for building, analyzing and ranking the possible scenarios. To cope with the issue of uncertain parameters of the environment in strategic planning, we use the concept of fuzzy set theory to enhance the proposed method. Finally the performance of the proposed method is illustrated in a strategic planning case in a pharmaceutical company.
\end{abstract}

*To whom all correspondence should be addressed.

\section{Introduction}

In today's world, the market climate changes more quickly and countries realize that globalization makes the world smaller and more competitive. Also customers seek products and services that can respond to their specific needs and firms attempt to create competitive advantages to keep their profit and market share. Complex business organizations and competitive environment uncertainty have focused business researchers' and practitioners' attention on the use of strategic planning to integrate and optimize management processes. These trends compel firms and countries to forecast future events and design a proper action for future trends.

Strategic planning helps an organization to cope with increasing environmental turbulence and complexity, more intense competitive pressures, and the pace of technological change (McDonald, 1992). The main focus of strategic planning is deciding what strategies should be used to create a sustainable competitive advantage for competing in a given product market.

So for making effective and sustained decisions in strategic planning in an uncertain environment, knowing the current condition of environment and forecasting the future trends of environmental factors is necessary and critical. The environment can be regarded as a number of different compartments and processes that interact in a complex system. The assessment of the environmental consequences of an event is therefore complicated by the variety of influencing factors (Andersson, Stjernstrom \& Fangmark, 2005). But because of rapid environmental changes, intensified competitiveness and uncertainty of environmental factors, making or formulating strategies based on traditional methods is become very difficult and in some cases impossible.

To cope with this issue in strategic planning, a popular tool is scenario analysis, whereby scenarios are built for possible events in future and strategies planned with respect to these scenarios.

A scenario is a description of a future situation and the course of events that enables one to progress from the original situation to the future situation. The word scenario is often abused, especially when used to describe any set of hypotheses. Of course, these hypotheses must simultaneously be pertinent, coherent, plausible, important, and transparent to meet all of desirable criteria (Godet, 2000). Qualitative scenarios describe possible futures in the form of words or symbols, while quantitative scenarios describe futures in numerical form (Alcamo, 2001). A good scenario should be relevant, consistent (coherent), probable and transparent. In principle, only a few substantially different scenarios are needed (Nguyen, De Kok \& Titus, 2006). The participatory approach to scenario building, which is widely acknowledged, requires a wide spectrum of knowledge and opinions from multidisciplinary team 
members (Schwab, Cerutti \& Von Reibnitz, 2003; Van der Heijden, 1996).

Scenario analysis has three main purposes. The first is to forecast the environment; the second is to evaluate strategic options, especially the robustness of them, against the possible scenarios; and the third is to provide a nontechnical audience a picture of future alternative states of the environment in an easily understandable form that can provide an effective format in which information in both qualitative and quantitative forms can be assimilated and represented (Derek \& Ahti, 1993; Nguyen et al., 2006).

But one problem is that scenarios build on experts' opinions and usually experts forecast future in linguistic expressions. So the knowledge and the experience of experts are often the data sources in strategic planning (Sarin, 1979). Another problem is the complexity associated with uncertain environmental factors and often lack of relevant historical data. The complexity of the environmental problems makes necessary the development and application of new tools capable of processing not only the numerical aspects, but also the experience of experts and wide public participation, which are all needed in the decision-making process. This paper aimed to develop a simple methodology to cope with this problem. The main contribution of this paper lies in the implementation of a simple methodology based on fuzzy set theory and fuzzy clustering aimed to assist decision makers in their strategic decision process.

The reason for using fuzzy set theory and fuzzy clustering stems from the complexity, uncertainty and lack of knowledge associated with environment. The fuzzy set theory is precisely a theory that provides a framework to handle the sources of uncertainty, including vagueness, ambiguity and imprecision, at the same time (Nguene \& Finger, 2007). Fuzzy set theory that was originally developed by Zadeh (1973), aims to formalize the linguistic reasoning in mathematical form, which provides a means of approximate characterization of phenomena that are too complex to be amenable to description in conventional quantitative terms.

Many papers have used fuzzy set theory in management issues like transportation (e.g. Sheu, 2005), logistics or supply chain (e.g. Wang \& Shu, 2007; Hu \& Sheu, 2003) and strategic management (e.g. Kardaras \& Karakostas, 1999), but few papers have applied fuzzy set theory to scenario analysis (Nguyen et al., 2006; Nguene \& Finger, 2007).
Using a fuzzy clustering approach in aggregating the possible scenarios into a few main scenarios and developing a method for calculating the consistency or compatibility of scenarios is what differentiates this research with the past works mentioned above.

The remainder of this paper is organized as follows. In Section 2 we describe the proposed methodology. For better understanding of the proposed method, a case study is presented in Section 3, and in Section 4 a summary of the work and some possible future works are presented.

\section{Methodology}

To cope with the issues of linguistic expression of an expert in strategic planning, uncertainties and lack of information about future forecasting, we use the concept of fuzzy set theory and fuzzy clustering to develop a hybrid method for scenario analysis. This method comprises five main steps:

Step 1 Defining key factors and describing their possible future trends in the opinion of experts.

Step 2 Generating all possible scenarios from a combination of factors' future trends.

Step 3 Calculating pair wise compatibility indexes and eliminating incompatible scenarios.

Step 4 Defining main scenarios with Fuzzy C-means clustering (FCM) method and ranking them by calculating the degree of possibility for each final scenario.

Step 5 Project the cluster centres for expressing and interpreting the main scenarios in linguistic terms.

The input of this system is qualitative expressions by several experts about key factors' future trends (step 1). Then these subjective qualitative expressions are translate into a quantitative form based on fuzzy numbers for generating all possible scenarios (steps 1 and 2). Scenario analysis and compatibility measurements are performed by fuzzy set theory operators (step 3) and by aggregating the possible compatible scenarios based on fuzzy clustering the main final scenarios are achieved. Finally, the main scenarios are presented and used in both quantitative and qualitative (linguistic expression) form. This process is illustrated in Figure 1.

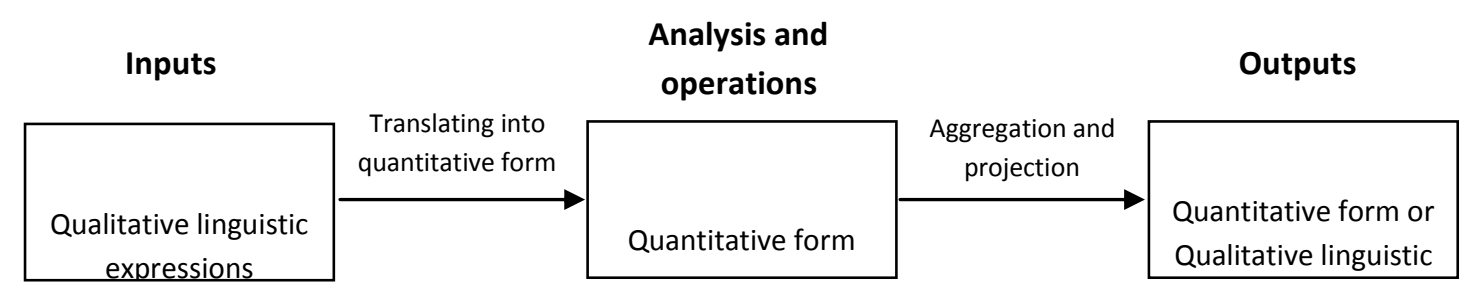

Figure 1: Methodology process 
The detail of each of the five steps is described in below.

\section{Defining the key factors and their future trends}

In this step, based on environmental analysis and experts' opinions, the most influential key factors (variables) are distilled. In strategic planning literature these factors often classified in five segments: Political, Economic, Cultural and Social, Technological, Demographic (Pearce \& Robinson, 2005; Mowen \& Minor, 1997). Consequently, we should define the main factors with respect to these areas and forecast their future trends by experts' opinions. But as mentioned earlier, one problem is that experts forecast the future in linguistic expressions (e.g. market demand for lifestyle drugs will be increased strongly). To cope with this issue we use fuzzy set theory. If the market demand is a linguistic variable, the value terms of this linguistic variable could be "low", "moderate" and "high" instead of crisp numbers. A linguistic expression like "market demand for lifestyle drugs will be high" could be modeled by a fuzzy number. A fuzzy number is a normal and convex fuzzy set with bounded support (Klir \& Yuan, 2002). The fuzzification, which can be described by the process of establishment of membership functions, requires several steps, consisting of the establishment of ranges in the numerical domains of the key factors concerned, the specification of boundaries in the fuzzy domains of associated fuzzy subsets and the selection of the shape of the membership functions (MFs) (Nguyen et al., 2006)". There are, in general, no rules for the selection of shape of a membership function when little data and expert's knowledge about a variable exist. Therefore, the symmetrically trapezoidal, triangular and Gaussian MFs are often chosen for this purpose. In this paper MFs of key factors have the triangular form (Figure 2).

In addition to the specification of the numerical ranges of variables, it is necessary to specify the boundaries of the associated fuzzy subsets. For example, from what value to what value can the market demand be considered to be "low" or "high".

The boundaries of fuzzy subsets may interred, i.e. one particular demand quantity can belong to both "low" and "'medium" fuzzy subsets (Figure 2). These boundaries are often established subjectively from the experience of experts.

\section{Generating all possible scenarios}

Now it is possible to generate the scenarios. Each combination of forecasted future trends of key factors makes a possible scenario. As illustrated in the case presented in Figure 3, the combination of forecasted trends makes 834 possible scenarios. So the number of scenarios grows very fast when the number of forecasted trends increases.

Many of these possible scenarios have a very low degree of possibility; we named them in this paper "incompatible scenarios". For a valid strategic planning, incompatible scenarios should be detected and eliminated. For this purpose, a method is proposed based on fuzzy set theory in the next section.

\section{Calculating the fuzzy compatibility index and eliminating incompatible scenarios}

One way to measure the degree of possibility of a scenario is to measure the compatibility of each pair of forecasted future trends in each scenario. However the relation between the pair wise forecasted trends often can not be clearly expressed by an expert, especially for long-term forecasting. Moreover there are no past data to refer to. Therefore, to determine the degree of possibility of a scenario that shows its importance, we define a fuzzy compatibility index (FCI) between each pair of forecasted future trends. The concept of triangular fuzzy number (TFN) is used for this purpose. As shown in Figure 4, five linguistic variables as TFNs between $\tilde{1}$ and $\widetilde{5}\left(\mathrm{FCI}_{(\mathrm{i}, \mathrm{j})}\right)$ were defined. FCIs were determined based on experts' opinions.

Experts determine the compatibility between each pair of forecasted trends in linguistic terms in a range of "very low" to "very high" (very low, low, medium, high and very high). For eliminating the incompatible scenarios two rules were used:

1. If one or more than one of FCIs between each pair of forecasted trends $\left(\mathrm{FCI}_{(\mathrm{i}, \mathrm{j})}\right)$ in a scenario is "very low" the related scenario was eliminated.

2. If the average of FCIs in a scenario is less than "Medium" (or fuzzy number $\tilde{3}$ ), the related scenario was eliminated.

At the end of this stage we have some compatible scenarios, but as mentioned before if there are too many scenarios (more than 5 or 6 ), they will lose their characteristics and blur the main issue. Therefore to solve this problem and to achieve the efficient number of scenarios, similar scenarios should be grouped. We use fuzzy clustering for this purpose as described in the next section.

\section{Defining the final scenarios and their ranking}

Classification methods can be divided in two main groups of techniques: discriminant analysis and cluster analysis. Cluster analysis refers to the unsupervised situation where little or no information is available about group structure prior to the classification. The goal is to find groups in the data. Discriminant analysis refers to situations where the membership of a set of samples is known and the main purpose is to build a classification rule applicable for new and unknown samples. 


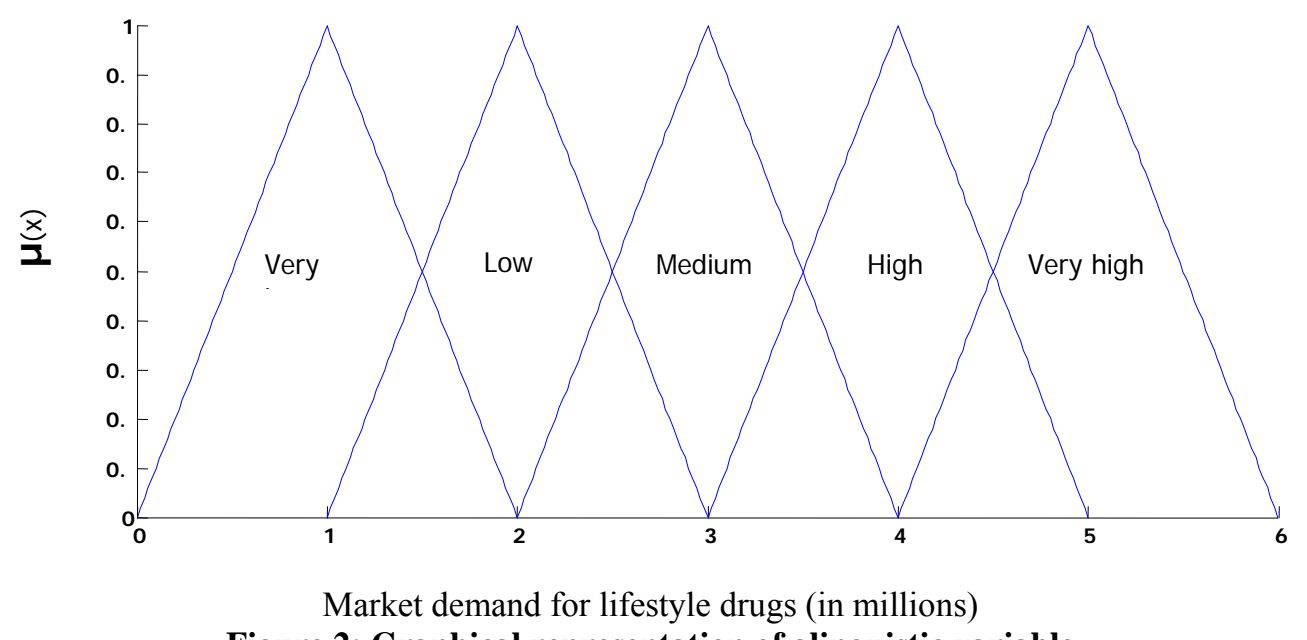

Figure 2: Graphical representation of alinguistic variable

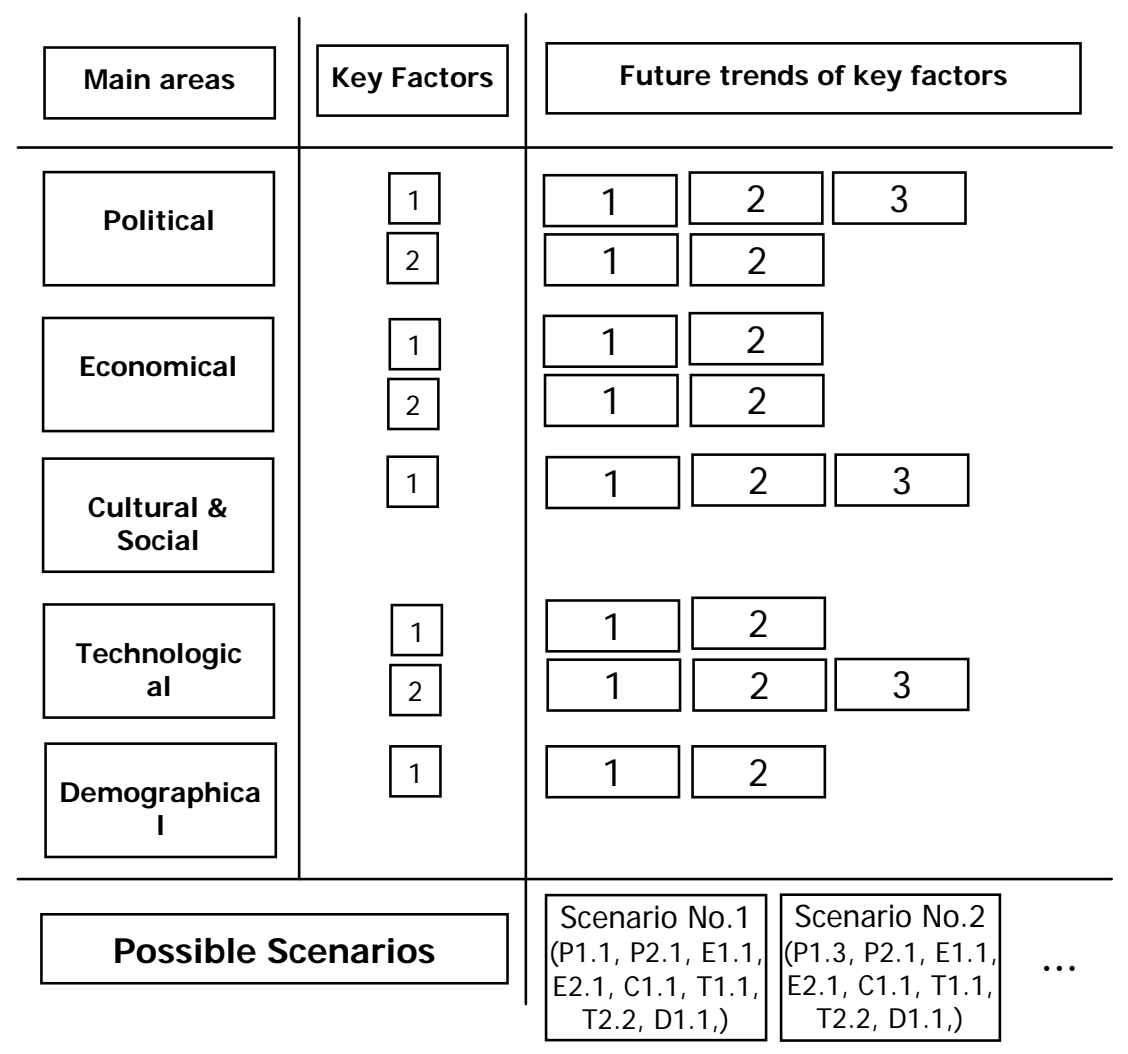

Figure 3: Generating possible scenarios

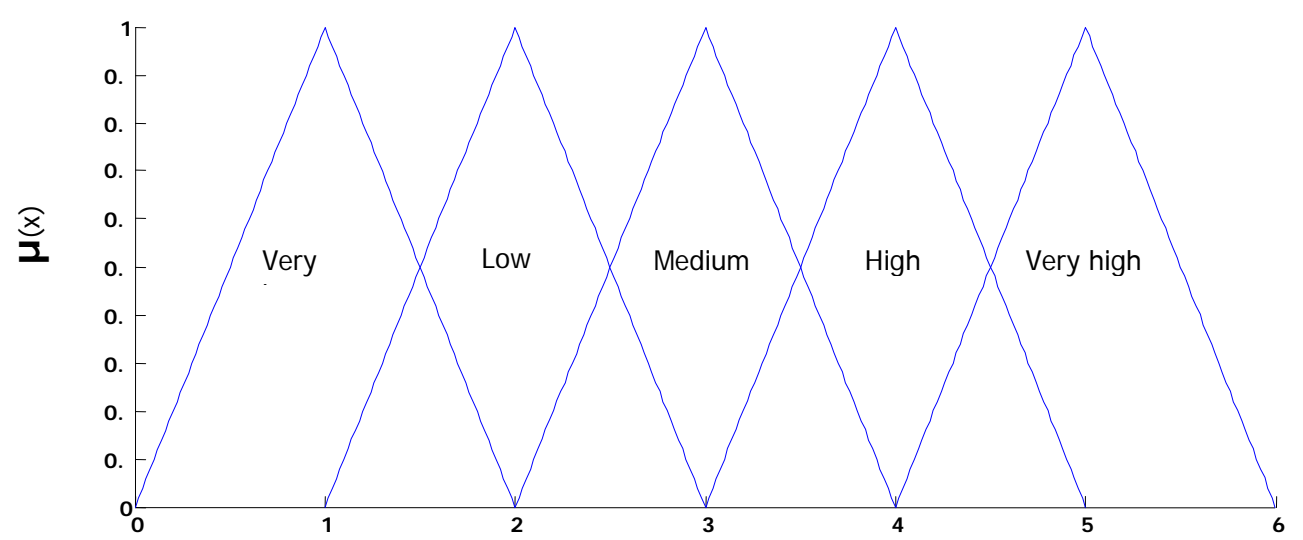

Figure 4: Linguistic variables for FCI 
Cluster analysis is based on partitioning a collection of data points into a number of subgroups, where the objects inside a cluster (a subgroup) show a certain degree of closeness or similarity. Hard clustering assigns each data point to one and only one of the clusters, with a degree of membership equal to one, assuming well defined boundaries between the clusters. This model does not often reflect the description of real data, where boundaries between subgroups might be fuzzy and where a more nuanced description of object affinity to the specific cluster is required (Gath \& Geva, 1989).

Fuzzy clustering, as an advanced clustering method, unlike the hard clustering methods, allows each data point to belong to several groups with different degrees of similarity bounded within the range of 0 and 1 . Another benefit is that in fuzzy clustering we can analyze multi-dimensional data consisting of linguistic attributes (Hu \& Sheu, 2003).

The Fuzzy C-means clustering algorithm (FCM) is based on the minimization of an objective function called C-means functional (Bezdek, 1975). The FCM algorithm uses a standard Euclidean distance norm, which induces hyper spherical clusters.

For the Fuzzy C-Means clustering algorithm there are three input parameters needed to run this function:

- The number of clusters or initializing partition matrix.

- The fuzziness weighting exponent.

- The maximum termination tolerance.

The two latter parameters have their default value, if they are not given by the user. The function calculates with the standard Euclidean distance norm, the norm inducing matrix at an $(n \times n)$ identity matrix. The result of the partition is collected in structure arrays.

In this paper we use FCM algorithm in our methodology to group the similar scenarios. The detail of the FCM algorithm is described in the appendix.

Another issue is determining the number of clusters that represent the number of final scenarios. It is important that we know how many clusters should be used in FCM method. For this purpose, a cluster validity method must be examined.

Validity methods began with Bezdek partition coefficient $\left(\mathrm{V}_{\mathrm{PC}}\right)$ and partition entropy $\left(\mathrm{V}_{\mathrm{PE}}\right)$ of $\mathrm{U}$ matrix, which shows the degree of membership of each data to each cluster. In this paper we use a more recent index known as the Fukuyama-Sugeno index. This index provided better response versus other validity indexes include partition coefficient and partition entropy of Bezdek (Pal \& Bezdek, 1994). The index is presented in the appendix. To choose the optimal number of clusters that shows the number of final scenarios, we proposed the below procedure.
Number of clusters $\mathrm{NC}=1$

Maximum allowed number of scenarios $\mathrm{MN}=\max (\mathrm{MN}$ is a case based number usually between 5 to 7 )

$\mathrm{FSI}_{1}=0$

While $\mathrm{FSI}_{(\mathrm{NC})}<\mathrm{FSI}_{(\mathrm{NC}-1)}$ and $\mathrm{NC}<\mathrm{MN}$

$\mathrm{NC}=\mathrm{NC}+1$

Calling FCM algorithm procedure

Calculating Fukuyama-Sugeno index $\mathrm{FSI}_{(\mathrm{NC})}$

After clustering the scenarios we have the main scenarios, but these scenarios do not have the same degree of possibility, because of the difference in their degree of possibility. To rank these main scenarios with respect to their importance (degree of possibility) we propose a formula as follow:

$\operatorname{RS}(\mathrm{h})=\frac{\sum_{\mathrm{k}=1}^{\mathrm{n}} \sum_{\mathrm{i}=1}^{\mathrm{m}} \sum_{\mathrm{j}=1}^{\mathrm{m}} \mu_{\mathrm{k}} \mathrm{FCI}_{(\mathrm{i}, \mathrm{j})}}{\mathrm{n}}$

$\mathbf{n}$ : number of compatible scenarios in each cluster.

m: number of forecasted future trends.

$\mu_{\mathrm{k}}$ : Degree of membership of scenario k to cluster $\mathrm{h}$.

This formula could properly represent the degree of possibility of the main scenarios. The scenarios that achieve a higher ranking score (RS) are more important than others, because the possibility of occurrence for these scenarios is higher than the others.

\section{Expressing and interpreting the main scenarios}

Ideally we should present the scenarios in linguistic expressions to senior management for decision making. To express the final scenarios in a linguistic statement we introduce a method base on the projection of cluster centres. In this method cluster centres were projected on each axis. Each axis is related to one key factor and as mentioned before we define linguistic variables on each axis base on fuzzy numbers. Therefore each cluster centre as a main scenario represents a scenario that includes all the key factors and their future trends. As a result we could explain each main scenario in linguistic expression with this method. In the next section the proposed method is used in a case study.

\section{Case study}

In this section the proposed method is applied to a case study in the pharmaceutical market. The studied case is a pharmaceutical Asian company with two plants and one research centre. The company is produces three drugs. Two of them are usual prescription drugs with an approximately smooth demand used in medical care activities. These drugs 
are under protection of government and direct government pricing rules. There is a possibility of exporting for these two drugs to two neighboring countries, which have a critical need for these drugs. But this opportunity is encountering some political obstacles. The other drug $(\mathrm{X})$ is an over-the-counter lifestyle drug without government protection and with free market pricing. Drug $\mathrm{X}$ is developed in the company's research centre and the company has patent rights to it. $X$ sales is almost $45 \%$ of the company's total revenues. One critical problem that threatens future $\mathrm{X}$ sales is the weakness of government laws in patent protection. This problem seems more important because now company decides to develop another lifestyle drug in its research center. The other critical factor is the size and structure of market demand. There are several forecasted future trends for this key factor because of environmental uncertainty. This has a significant effect on the company's strategic choice. If the share of lifestyle drugs in the structure of demand increases or decreases the strategic choice will change significantly. We define the MFs of each key factor base on expert opinions. For example the MF of "share of lifestyle drugs in total demand" is illustrated in Figure 5. Other key factors and their forecasted future trends are illustrated in Table 1.

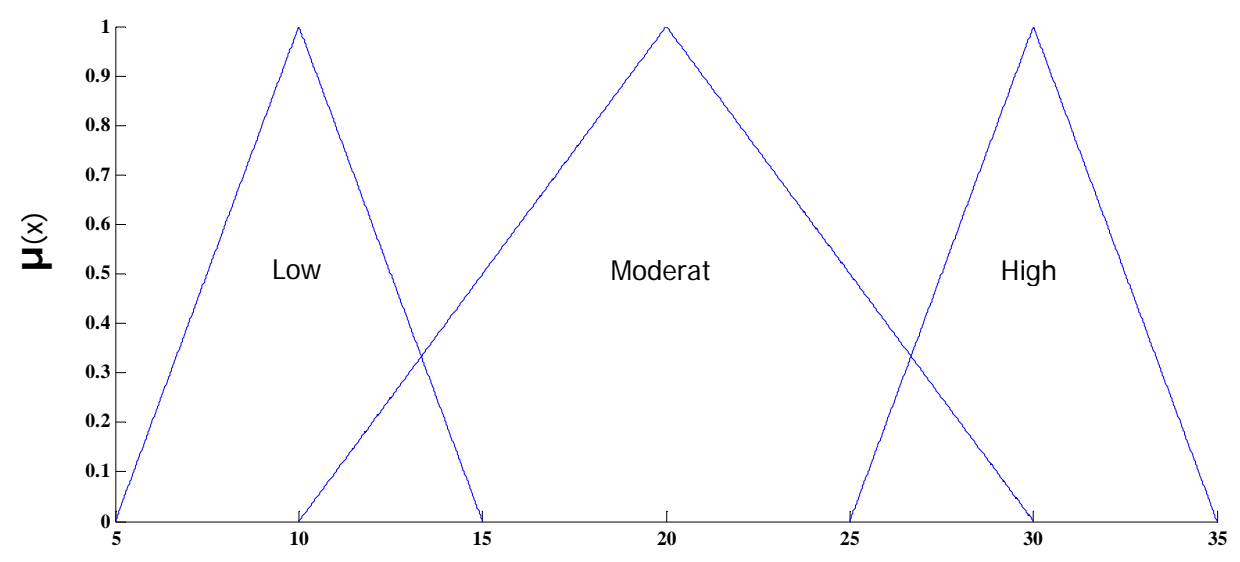

Figure 5: Share of lifestyle drugs in total demand (\%) MF

Table 1: Key factors in Pharmaceutical industry and their future trends determined by experts

\begin{tabular}{|c|c|c|c|c|c|c|}
\hline \multirow{2}{*}{ Main Areas } & \multirow{2}{*}{ Key factors } & \multicolumn{5}{|c|}{ Future trends } \\
\hline & & VL & $\mathbf{L}$ & $\mathbf{M}$ & $\mathbf{H}$ & VH \\
\hline \multirow{2}{*}{ Political-Legal } & P1. Possibility of export to neighbor countries & & $\bullet$ & & $\bullet$ & \\
\hline & P2. Extension of health care insurance by Gov. & & & $\bullet$ & & $\bullet$ \\
\hline \multirow{3}{*}{ Economical } & E1. Rate of merging in Pharmaceutical industry & & $\bullet$ & & $\bullet$ & \\
\hline & E2. Share of lifestyle drugs in total demand & & $\bullet$ & $\bullet$ & $\bullet$ & \\
\hline & E3. Total demand & & & $\bullet$ & & $\bullet$ \\
\hline Cultural-Social & C1. Culture of consuming lifestyle drugs & & $\bullet$ & & $\bullet$ & \\
\hline Demographical & D1. Population Senility & & & & $\bullet$ & \\
\hline
\end{tabular}


Table 2: All possible scenarios

\begin{tabular}{|c|c|c|c|c|c|c|c|c|c|}
\hline \multirow{2}{*}{$\begin{array}{c}\text { Scenario } \\
\text { No. }\end{array}$} & \multicolumn{9}{|c|}{ Forecasted trends } \\
\hline & P1.2 & P2.2 & E1.1 & E2.1 & E2.2 & E2.3 & E3.1 & C1.1 & D1.1 \\
\hline 1 & $\mathrm{P} 1.1$ & $\mathrm{P} 2.1$ & E1.1 & & E2.1 & & E3.1 & $\mathrm{C} 1.1$ & D1.1 \\
\hline 2 & $\mathrm{P} 1.2$ & $\mathrm{P} 2.1$ & E1.1 & & E2.1 & & E3.1 & $\mathrm{C} 1.1$ & D1.1 \\
\hline . & . & . & . & & . & & . & . & . \\
\hline . & . & . & . & & . & & . & . & . \\
\hline . & . & . & . & & . & & . & . & . \\
\hline 46 & $\mathrm{P} 1.2$ & P2.1 & E1.1 & & E2.3 & & E3.2 & $\mathrm{C} 1.2$ & D1.1 \\
\hline 47 & P1.1 & P2.1 & E1.2 & & E2.3 & & $\mathrm{E} 3.2$ & $\mathrm{C} 1.2$ & D1.1 \\
\hline 48 & $\mathrm{P} 1.2$ & P2.1 & E1.2 & & E2.3 & & $\mathrm{E} 3.2$ & $\mathrm{C} 1.2$ & D1.1 \\
\hline . & . & . & . & & . & & . & . & . \\
\hline . & . & . & . & & . & & . & . & . \\
\hline . & . & . & . & & . & & . & . & . \\
\hline 95 & P1.1 & P2.2 & E1.2 & & E2.3 & & E3.2 & C1.2 & D1.1 \\
\hline 96 & P1.2 & $\mathrm{P} 2.2$ & E1.2 & & E2.3 & & E3.2 & $\mathrm{C} 1.2$ & D1.1 \\
\hline
\end{tabular}

Table 3: The pairwise FCIs between forecasted future trends

\begin{tabular}{c|c|c|c|c|c|c|c|c|c|c|c|c|c|c} 
& P1.1 & P1.2 & P2.1 & P2.2 & E1.1 & E1.2 & E2.1 & E2.2 & E2.3 & E3.1 & E3.2 & C1.1 & C1.2 & D1.1 \\
\hline P1.1 & & & $\widetilde{3}$ & $\widetilde{3}$ & $\widetilde{3}$ & $\widetilde{3}$ & $\widetilde{2}$ & $\widetilde{3}$ & $\widetilde{4}$ & $\widetilde{3}$ & $\widetilde{1}$ & $\widetilde{3}$ & $\widetilde{3}$ & $\widetilde{3}$ \\
\hline P1.2 & & & $\widetilde{3}$ & $\widetilde{3}$ & $\widetilde{3}$ & $\widetilde{4}$ & $\widetilde{4}$ & $\widetilde{3}$ & $\widetilde{2}$ & $\widetilde{1}$ & $\widetilde{5}$ & $\widetilde{3}$ & $\widetilde{3}$ & $\widetilde{3}$ \\
\hline P2.1 & & & & $\widetilde{3}$ & $\widetilde{3}$ & $\widetilde{3}$ & $\widetilde{3}$ & $\widetilde{3}$ & $\widetilde{4}$ & $\widetilde{3}$ & $\widetilde{3}$ & $\widetilde{3}$ & $\widetilde{3}$ \\
\hline P2.2 & & & & & $\widetilde{3}$ & $\widetilde{4}$ & $\widetilde{4}$ & $\widetilde{3}$ & $\widetilde{2}$ & $\widetilde{3}$ & $\widetilde{4}$ & $\widetilde{3}$ & $\widetilde{3}$ & $\widetilde{4}$ \\
\hline E1.1 & & & & & & $\widetilde{3}$ & $\widetilde{3}$ & $\widetilde{2}$ & $\widetilde{3}$ & $\widetilde{3}$ & $\widetilde{3}$ & $\widetilde{2}$ & $\widetilde{3}$ \\
\hline E1.2 & & & & & & $\widetilde{3}$ & $\widetilde{3}$ & $\widetilde{4}$ & $\widetilde{3}$ & $\widetilde{4}$ & $\widetilde{3}$ & $\widetilde{4}$ & $\widetilde{3}$ \\
\hline E2.1 & & & & & & & & & & $\widetilde{2}$ & $\widetilde{4}$ & $\widetilde{4}$ & $\widetilde{1}$ & $\widetilde{2}$ \\
\hline E2.2 & & & & & & & & & $\widetilde{3}$ & $\widetilde{3}$ & $\widetilde{3}$ & $\widetilde{3}$ & $\widetilde{3}$ \\
\hline E2.3 & & & & & & & & & $\widetilde{4}$ & $\widetilde{2}$ & $\widetilde{2}$ & $\widetilde{4}$ & $\widetilde{4}$ \\
\hline E3.1 & & & & & & & & & & & & $\widetilde{3}$ & $\widetilde{3}$ & $\widetilde{3}$ \\
\hline E3.2 & & & & & & & & & & & $\widetilde{2}$ & $\widetilde{4}$ & $\widetilde{4}$ \\
\hline C1.1 & & & & & & & & & & & & & & $\widetilde{2}$ \\
\hline C1.2 & & & & & & & & & & & & & & $\widetilde{4}$ \\
\hline D1.1 & & & & & & & & & & & & & & \\
\hline
\end{tabular}

As explained before, now all possible scenarios must be generated from the combination of forecasted future trends. In this case with respect to Table 1 , the combination of forecasted trends makes 96 possible scenarios (see Table 2). To omit the incompatible scenarios, first FCIs between forecasted trends should be determined based on experts' opinions. Pairwise FICs for the pharmaceutical company case are illustrated in Table 3. After determining FCIs, we can now eliminate the incompatible scenarios using the two rules proposed in Section 2.3.

After exerting the rules on possible scenarios of the studied case, 35 scenarios remained (see Table 4). But as mentioned before, for a good scenario analysis the number of scenarios should not exceed from 6, otherwise they will lose their characteristics and blur the main issue. Therefore, as described in Section 2.4 we use the FCM fuzzy clustering method to group the similar scenarios. The results of clustering are shown in Table 5. The optimum number of clusters that represent the number of final main scenarios in this case is 5 . As mentioned before this number is calculated on the basis of the Fukuyama-Sugeno validity index. Now we can project the cluster centres to present the final main scenarios in linguistic terms. Final main scenarios and their related ranking scores are shown in Table 6. Ranking scores show the degree of possibility of each scenario. 
Table 4: Eliminating the incompatible scenarios

\begin{tabular}{|c|c|c|c|c|c|c|c|}
\hline Scenario No. & $\begin{array}{c}\text { Scenario } \\
\text { Value }\end{array}$ & $\begin{array}{c}\text { Elimination } \\
\text { Rule 1 }\end{array}$ & $\begin{array}{c}\text { Elimination } \\
\text { Rule } 2\end{array}$ & Scenario No. & $\begin{array}{c}\text { Scenario } \\
\text { Value }\end{array}$ & $\begin{array}{c}\text { Elimination } \\
\text { Rule 1 }\end{array}$ & $\begin{array}{c}\text { Elimination } \\
\text { Rule } 2\end{array}$ \\
\hline 1 & 2,904 & not rejected & rejected & 49 & 2,952 & not rejected & rejected \\
\hline 2 & 2,904 & rejected & rejected & 50 & 2,952 & rejected & rejected \\
\hline 3 & 2,952 & not rejected & rejected & 51 & 3 & not rejected & not rejected \\
\hline 4 & 3,142 & rejected & not rejected & 52 & 3,047 & rejected & not rejected \\
\hline 5 & 2,85 & rejected & rejected & 53 & 3 & rejected & not rejected \\
\hline 6 & 3,14 & not rejected & not rejected & 54 & 3,285 & not rejected & not rejected \\
\hline 7 & 2,904 & rejected & rejected & 55 & 3,095 & rejected & not rejected \\
\hline 8 & 3,238 & not rejected & not rejected & 56 & 3,428 & not rejected & not rejected \\
\hline 9 & 2,809 & rejected & rejected & 57 & 2,857 & rejected & rejected \\
\hline 10 & 2,809 & rejected & rejected & 58 & 2,857 & rejected & rejected \\
\hline 11 & 2,904 & rejected & rejected & 59 & 3 & rejected & not rejected \\
\hline 12 & 2,952 & rejected & rejected & 60 & 3,047 & rejected & not rejected \\
\hline 13 & 2,857 & rejected & rejected & 61 & 3 & rejected & not rejected \\
\hline 14 & 3,142 & rejected & not rejected & 62 & 3,285 & rejected & not rejected \\
\hline 15 & 3 & rejected & not rejected & 63 & 3,190 & rejected & not rejected \\
\hline 16 & 3,333 & rejected & not rejected & 64 & 3,523 & rejected & not rejected \\
\hline 17 & 3 & not rejected & not rejected & 65 & 3 & not rejected & not rejected \\
\hline 18 & 2,904 & rejected & rejected & 66 & 2,904 & rejected & rejected \\
\hline 19 & 3 & not rejected & not rejected & 67 & 3,047 & not rejected & not rejected \\
\hline 20 & 2,952 & rejected & rejected & 68 & 3 & rejected & not rejected \\
\hline 21 & 2,857 & rejected & rejected & 69 & 2,952 & rejected & rejected \\
\hline 22 & 3,047 & not rejected & not rejected & 70 & 3,142 & not rejected & not rejected \\
\hline 23 & 2,904 & rejected & rejected & 71 & 3,047 & rejected & not rejected \\
\hline 24 & 3,142 & not rejected & not rejected & 72 & 3,285 & not rejected & not rejected \\
\hline 25 & 3,047 & not rejected & not rejected & 73 & 3,047 & not rejected & not rejected \\
\hline 26 & 2,952 & rejected & rejected & 74 & 2,952 & rejected & rejected \\
\hline 27 & 3,142 & not rejected & not rejected & 75 & 3,190 & not rejected & not rejected \\
\hline 28 & 3,095 & rejected & not rejected & 76 & 3,142 & rejected & not rejected \\
\hline 29 & 3 & rejected & not rejected & 77 & 3,095 & rejected & not rejected \\
\hline 30 & 3,190 & not rejected & not rejected & 78 & 3,285 & not rejected & not rejected \\
\hline 31 & 3,142 & rejected & not rejected & 79 & 3,285 & rejected & not rejected \\
\hline 32 & 3,523 & not rejected & not rejected & 80 & 3,523 & not rejected & not rejected \\
\hline 33 & 3,047 & not rejected & not rejected & 81 & 3 & not rejected & not rejected \\
\hline 34 & 2,857 & rejected & rejected & 82 & 2,809 & rejected & rejected \\
\hline 35 & 3,095 & not rejected & not rejected & 83 & 3,142 & not rejected & not rejected \\
\hline 36 & 2,9045 & rejected & rejected & 84 & 3 & rejected & not rejected \\
\hline 37 & 2,809 & rejected & rejected & 85 & 2,857 & rejected & rejected \\
\hline 38 & 2,904 & not rejected & rejected & 86 & 2,952 & not rejected & rejected \\
\hline 39 & 2,952 & rejected & rejected & 87 & 3,047 & rejected & not rejected \\
\hline 40 & 3,095 & not rejected & not rejected & 88 & 3,190 & not rejected & not rejected \\
\hline 41 & 3,190 & not rejected & not rejected & 89 & 3,142 & not rejected & not rejected \\
\hline 42 & 3 & rejected & not rejected & 90 & 2,952 & rejected & rejected \\
\hline 43 & 3,380 & not rejected & not rejected & 91 & 3,380 & not rejected & not rejected \\
\hline 44 & 3,238 & rejected & not rejected & 92 & 3,238 & rejected & not rejected \\
\hline 45 & 3,047 & rejected & not rejected & 93 & 3,095 & rejected & not rejected \\
\hline 46 & 3,142 & not rejected & not rejected & 94 & 3,190 & not rejected & not rejected \\
\hline 47 & 3,285 & rejected & not rejected & 95 & 3,380 & rejected & not rejected \\
\hline 48 & 3,428 & not rejected & not rejected & 96 & 3,523 & not rejected & not rejected \\
\hline
\end{tabular}


Table 5: Cluster centres

\begin{tabular}{cccccccc}
\hline $\begin{array}{c}\text { Cluster } \\
\text { centres }\end{array}$ & P1 & P2 & E1 & E2 & E3 & C1 & D1 \\
\hline No.1 & 3.2109 & 94.9015 & 29.7549 & 29.7424 & 11.7109 & 3.4991 & 40 \\
No.2 & 3.0092 & 94.8687 & 29.6747 & 14.1130 & 11.5092 & 2.4952 & 40 \\
No.3 & 2.5632 & 60.0398 & 29.7551 & 22.4906 & 11.4632 & 2.8239 & 40 \\
No.4 & 2.5603 & 94.9044 & 10.0680 & 22.3129 & 11.4603 & 3.0363 & 40 \\
No.5 & 2.5629 & 60.0536 & 10.2252 & 22.5126 & 11.4629 & 3.0328 & 40 \\
\hline
\end{tabular}

Table 6: The final main scenarios and their ranking score

\begin{tabular}{ccccccccc}
\hline $\begin{array}{c}\text { Main Final } \\
\text { Scenarios }\end{array}$ & P1 & P2 & E1 & E2 & E3 & C1 & D1 & RS \\
\hline No.1 & Medium & High & High & High & High & High & High & 21.0132 \\
No.2 & Medium & High & High & Low & Medium & Low & High & 19.3064 \\
No.3 & Low & Medium & High & Medium & Medium & Medium & High & 24.5509 \\
No.4 & Low & High & Low & Medium & Medium & Medium & High & 22.7351 \\
No.5 & Low & Medium & Low & Medium & Medium & Medium & High & 24.0394 \\
\hline
\end{tabular}

Results of clustering show that in this case, five main scenarios could explain the possible future situation in the Pharmaceutical market. As is shown in Table 6 scenario No.3 is the most possible scenario. We could explain main scenario No.3 in a linguistic statement as follow.

There is a low possibility of exporting to two neighboring countries and the health care insurance will be extended moderately. Pharmaceutical companies will merge, especially with regard to research laboratories. Population senility will grow highly. Total demand, share of lifestyle drugs and the culture of consuming lifestyle drugs will grow moderately.

\section{Conclusion}

Scenario analysis is a powerful tool to cope with environment changes in strategic planning. But it associates with two problems, one is that scenarios build on expert's opinions and almost experts forecast future in linguistic expressions and second is the complexity associated with uncertain environmental factors and often lack of relevant historical data.

In this paper we introduce a method for scenario analysis in strategic planning to cope with the issues of uncertain parameters of environment and linguistic expression of an expert in strategic planning. We use fuzzy set theory and fuzzy clustering method to represent and group the expert's linguistic expressions. The proposed method is used for scenario analysis in a case in Pharmaceutical market to illustrate the performance. At the end we propose the following issues for future researches.

- Using the fuzzy clustering method that works with fuzzy data.
- $\quad$ Using fuzzy multi criteria decision making (FMCDM) methods for scenario ranking.

- $\quad$ Relation of scenario analysis and strategy ranking.

- Relation of scenario analysis and the robustness of strategies.

\section{References}

Alcamo, J. 2001. Scenarios as tools for international environmental assessments. Environmental Issue Report, No. 24. Experts' Corner Report. Prospects and Scenarios No. 5. Copenhagen, Denmark: European Environment Agency.

Andersson, A.S., Stjernstrom, O. \& Fangmark, I. 2005. 'Use of questionnaires and an expert panel to judge the environmental consequences of chemical spills for the development of an environment-accident index', Journal of Environmental Management, 75: 247-261.

Bezdek, J.C. \& Dunn, J.C. 1975. 'Optimal fuzzy partitions: A heuristic for estimating the parameters in a mixture of normal distributions', IEEE Trans. Comp. C-24:835-838.

Bezdek, J.C. 1981. Pattern recognition with fuzzy objective function algorithms. New York: Plenum Press.

Derek, W.B. \& Athi, A.S. 1993. 'Forecasting with scenarios', European Journal of Operational Research, 68:291-303.

Hu, T.L. \& Sheu, J.B. 2003. 'A fuzzy-based customer classification method for demand-responsive logistical distribution operations', Fuzzy Sets and Systems, 139(2):431-450. 
Kardaras, D. \& Karakostas, B. 1999.'The use of fuzzy cognitive maps to simulate the information systems strategic planning process', Information and Software Technology, 41:197-210

Klir, G.J. \& Yuan, B. 2002. Fuzzy sets and fuzzy logic theory and application. India: Prentice Hall of India.

Gath, I. \& Geva, A.B. 1989. 'Unsupervised optimal fuzzy clustering', IEEE Trans. Pattern Anal. Machine Intell. PAM 1-11(7):773-781.

Godet, M. 2000. 'The art of scenarios and strategic planning: Tools and pitfalls', Technological Forecasting and Social Change, 65:3-22.

McDonald, M.H.B. 1992. 'Strategic marketing planning: A state-of-the-art review', Marketing Intelligence and Planning, 10:4-22.

McDonald, M. 1996. 'Strategic marketing planning: Theory, practice and research agendas', Journal of Marketing Management, 12: 5-27.

Mowen J.C. \& Minor, M.J. 1997. Consumer behavior. New York, Prentice-Hall.

Nguene, G.N. \& Finger, M. 2007. 'A fuzzy-based approach for strategic choices in electric energy supply: The case of a Swiss power provider on the eve of electricity market opening, Engineering Applications of Artificial Intelligence, 20:37-48.

Nguyen, T.G., De Kok, J.L. \& Titus, M.J. 2006. 'A new approach to testing an integrated water systems model using qualitative scenarios', Environmental Modelling \& Software, 22(11):1557-1571.

Pal, N.R. \& Bezdek, J.C. 1994. 'On cluster validity for Fuzzy c-Means Model'. Submitted to IEEE Transactions on Fuzzy Systems.

Pearce, L.A. \& Robinson R.B. 2005. Strategic management: Formulation, implementation and control. NewYork, McGraw-Hill.

Sarin, R.K. 1979. 'An approach to cross impact analysis', Futures, 10:543-554.

Schwab, P., Cerutti, F. \& Von Reibnitz, C. 2003. 'Foresightusing scenarios to shape the future of agriculture research', Foresight 5(1):55e61.

Sheu, J.B. 2005. 'A fuzzy clustering approach to real-time demand-responsive bus dispatching', Fuzzy Sets and Systems, 15: 437-455.

Subhash, J.C. 1990. Marketing planning and strategy. $3^{\text {rd }}$ Edition. Cincinnati, OH: South-Western Publishing Co.

Van der Heijden, K. 1996. Scenarios: The art of strategic conversation. Chichester: John Wiley \& Sons.
Wang, J. \& Shu Y. 2007. 'A possibilistic decision model for new product supply chain design', European Journal of Operational Research 177: 1044-1061.

Wang, H.F. 1999. 'A fuzzy approach to scenario analysis in strategic planning'. In IEEE International Fuzzy Systems Conference Proceedings, Seoul, Korea.

Zadeh L.A. 1973. 'Outline of a new approach to the analysis of complex systems and decision processes', IEEE Transactions on Systems, Man, Cybernetics, SMC-3, $28 \mathrm{e} 34$. 


\section{Appendix}

\section{FCM Clustering Algorithm}

Given the data set $\mathrm{X}$ which includes $\mathrm{X}$ and $\mathrm{Y}$, the number of clusters $1<\mathrm{c}<\mathrm{N}$, the weighting exponent $\mathrm{m}>1$, the termination tolerance $\varepsilon>0$ and the norm-inducing matrix $A$, the algorithm tracks the following steps.

Step 1: Calculate the cluster centers

$\mathrm{V}_{\mathrm{i}}^{(1)}=\frac{\sum_{\mathrm{k}=1}^{\mathrm{N}}\left(\mu_{\mathrm{ik}}^{(1-1)}\right)^{\mathrm{m}} \mathrm{x}_{\mathrm{k}}}{\sum_{\mathrm{k}=1}^{\mathrm{N}}\left(\mu_{\mathrm{i}, \mathrm{k}}^{(1-1)}\right)^{\mathrm{m}}}, 1 \leq \mathrm{i} \leq \mathrm{c}$

Step 2: Compute the distances:

$D_{i k A}^{2}=\left(x_{k}-v_{i}\right)^{T} A\left(x_{k}-v_{s}\right), 1 \leq i \leq c, 1 \leq k \leq N$

Step 3: Update the partition matrix:

$\mu_{\mathrm{i}, \mathrm{k}}^{(1)}=\frac{1}{\sum_{\mathrm{j}=1}^{\mathrm{c}}\left(\mathrm{D}_{\mathrm{ikA}} / \mathrm{D}_{\mathrm{jkA}}\right)^{2 /(m-1)}}$

This steps will be repeated for $1=1,2, \ldots$ until

$\left\|\mathrm{U}^{(1)}-\mathrm{U}^{(1-1)}\right\|<\varepsilon$

\section{Fukuyama-Sugeno validity index}

Let $\mathrm{U} \in \mathrm{Mfc}$ and $\mathrm{v}=\left(\mathrm{v}_{1}, \mathrm{v}_{2}, \ldots, \mathrm{v}_{\mathrm{c}}\right)$ be vector of distinct points vi $\in \mathrm{R}^{\mathrm{p}}$ for $1 \leq \mathrm{i} \leq \mathrm{C}$ (here they are cluster centers). Fukuyama and Sugeno presents a new cluster validity index $\left(\mathbf{V}_{\mathrm{FS}}\right)$ as follows:

$\boldsymbol{V}_{\mathrm{FS}}(U, V ; X)=\sum_{\mathrm{i}=1: \mathrm{C}} \sum_{\mathrm{k}=1: \mathrm{N}}\left(\mathrm{U}_{\mathrm{i}, \mathrm{k}}\right)^{\mathrm{m}}\left(\left\|\mathrm{x}_{\mathrm{k}}-\mathrm{v}_{\mathrm{i}}\right\|^{2}-\left\|\mathrm{v}_{\mathrm{i}}-\overline{\mathrm{v}}\right\|^{2}\right)$

A good $(U, V)$ pair should produce a small value of the index. 